\title{
Variability of Transionospheric Signal Time Delay at Subauroral Latitudes
}

\author{
HAIM SOICHER
}

\begin{abstract}
Faraday observations were conducted at Anchorage, AK $\left(61.04^{\circ} \mathrm{N}, 149.75^{\circ} \mathrm{W}\right)$, utilizing beacon transmissions from a geostationary satellite during the period just following the minimum phase of solar cycle 21 , and, for comparison purpose, during a short period following the maximum phase of that same cycle. Near the minimum phase average maximum monthly values of total electron content (TEC), which is proportional to transionospheric signal time delay, were below $15 \times 10^{16}$ $\mathrm{el} \mathrm{m}^{-2}$, while individual daily maximum values never exceeded $20 \times 10^{16}$ el $\mathrm{m}^{-2}$. Near the maximum phase TEC average monthly values were larger by a factor of two. Seasonal and day-to-day variabilities were observed. Unique representation of the data has permitted the study of day-to-day variability of TEC. For example, near minimum phase during all seasons the TEC structure appears uniform from day to day during the buildup and decay phases of the local ionosphere. During the maximum and minimum of the diurnal phase, the TEC structure variability is seasonally dependent. During periods of magnetic sudden commencements, which rarely occurred in the observation periods, significant positive phase responses of TEC did not materialize near solar minimum, but were correlated near solar maximum.
\end{abstract}

\section{INTRODUCTION}

A SPACE-BASED radio navigation system could provide military and civilian users with precise three-dimensional position and velocity data. To determine his position and velocity, the user will cross correlate the coded time signal received from a satellite with the same coded time signal generated in his receiver. The phase difference, or equivalently the time displacement, between the incoming phase and user receiver's phase determines the range to the satellite. Simultaneous measurements of such relative phases from four different satellites will permit the user to determine his range and his clock bias with respect to the satellite's position and clock, respectively.

This range from the user to the various satellites is, of course, not the geometric range. A propagating signal is slowed down by an amount directly proportional to total electron content (TEC) along its path, and inversely proportional to the square of the operating frequency [1].

The total signal delay time (at VHF and above) $t(\mathrm{~s})$ is

$$
t=1 / c \int_{0}^{s} d s+40.3 / c f^{2} \int_{0}^{s} N d s
$$

where $d s$ is an element of distance along the signal's path, $f$ is the operating frequency in $\mathrm{Hz}, c$ is the speed of light in $\mathrm{m} / \mathrm{s}, N$

Manuscript received November 5, 1985; revised April 18, 1986.

The author is with the Communications/Automatic Data Processing Center, U.S. Army Communications-Electronics Command, Fort Monmouth, NJ 07703 .

IEEE Log Number 8610019 is the electron density per meter cubed, and $O$ and $S$ are the observer and satellite positions, respectively.

The first term in (1) represents the transit time of the signal in free space, whereas the second term represents the excess time delay of the signal along the path. While the ionospheric delay may be mitigated by the use of two frequency systems, their expense and other constraints may negate their use. A determination of TEC variability is thus necessary, for which the systems must compensate or adapt.

The Faraday polarization rotation technique has been used to obtain TEC data. A plane polarized wave propagating in the anisotropic ionosphere may be regarded as the vector sum of the ordinary and extraordinary magneto-ionic components. Since the two components travel at different phase velocities, the plane of the polarization rotates continuously along the signal's path. The total rotation from a satellite signal source to observer is directly proportional to TEC.

Faraday rotation observations were conducted at Anchorage, AK $\left(61.04^{\circ} \mathrm{N}, 149.75^{\circ} \mathrm{W}\right)$, using the signal of a geostationary satellite located at $96^{\circ} \mathrm{W}$. The geographic subionospheric point (i.e., the coordinates of the point at which the signal path intersects a mean ionospheric altitude of $420 \mathrm{~km}$ ) is $52.1^{\circ} \mathrm{N}, 131.0^{\circ} \mathrm{W}$; the invariant latitude is $56.9^{\circ}$; the magnetic dip is $71.9^{\circ}$; and the $L$ shell value is 3.35 . The ionospheric characteristics observed are considered to be those of which are prevalent at the subionospheric point rather than those at the location of the receiving apparatus. The beacon frequency of the satellite was $136.38 \mathrm{MHz}$.

The receiving equipment was composed of an electronically rotated polarimeter which compares the left and right circular polarization components of the incident linear polarization on an eight-element crossed Yagi antenna [2]. The recording of the polarimeter output is done in analog form on a chart recorder. The conversion of polarization rotation to total electron content is accomplished by standard procedures, at 15 min intervals, taking into account the initial polarization transmission at the satellite source and antenna polarization calibration [3].

The transmission from the satellite is continuous except for an occasional shutoff of the satellite for power conservation purposes.

\section{TEC Structure}

At midlatitudes the primary source of ionization is solar EUV and UV. Day-to-day variations in TEC reflect changes in the neutral atmosphere and winds. At higher latitudes, the magnetosphere-ionosphere interaction is a dominant dynamic force in addition to solar ionization, thus contributing to 


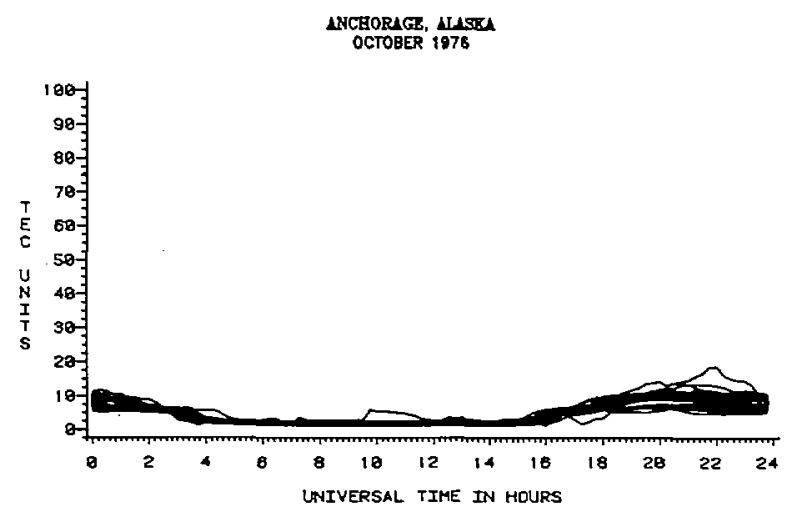

(a)

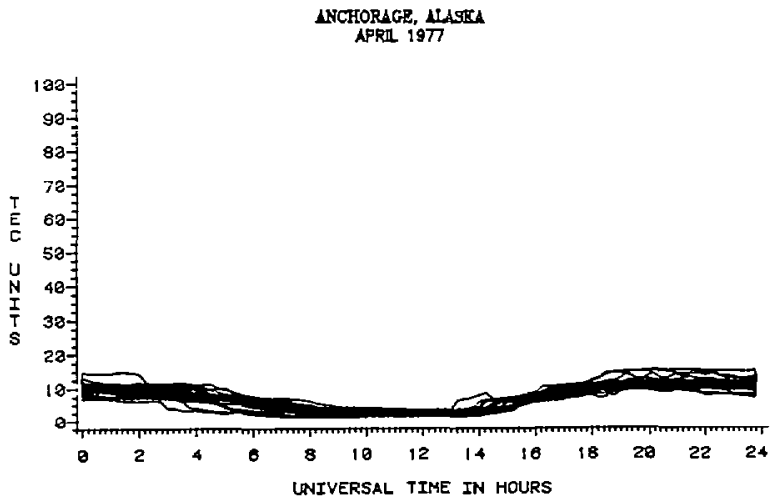

(c)

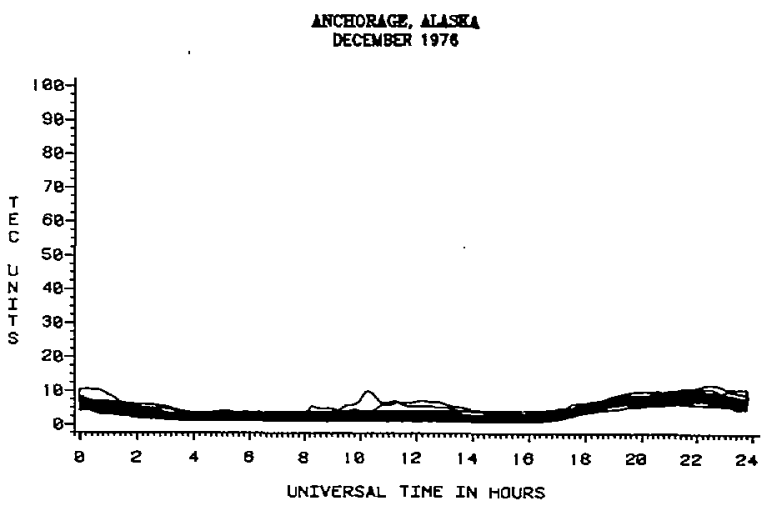

(b)

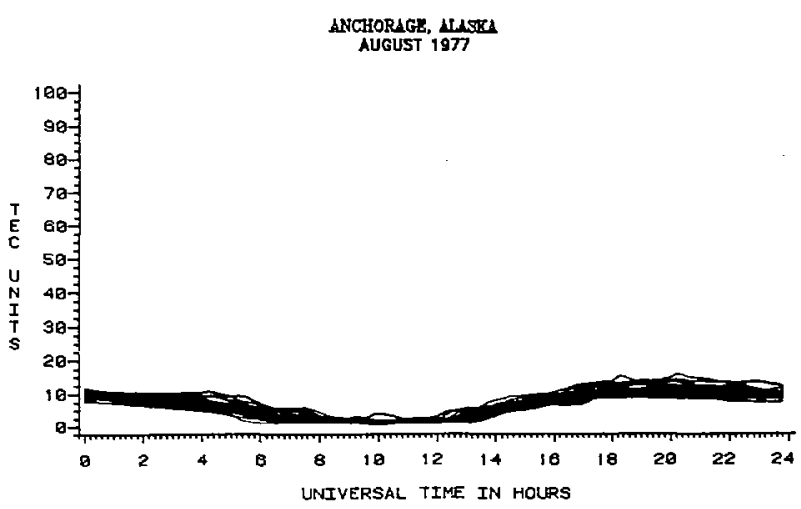

(d)

Fig. 1. Superposed diurnal variations of total electron content in Anchorage, AK. (a) October 1976. (b) December 1976. (c) April 1977. (d) August 1977. Ordinate: 1 TEC unit $-10^{16} \mathrm{el} \mathrm{m}^{-2}$; abscissa: time in UT.

particular aspects of TEC variability which are not directly correlated with solar flux variations.

The superposed diurnal variation of TEC grouped in monthly intervals for the seasonally representative months of October and December 1976 and April and August 1977, are shown in Figs. 1 and 2. The smoothed observed sunspot numbers were $13,15,22,33$ respectively, which were near the minimum of sunspot cycle 21 (12) which occurred in March 1976. The TEC values are normalized to the vertical direction, and have the unit of $10^{16} \mathrm{el} \mathrm{m}^{-2}$ (a TEC unit $=10^{16}$ $\mathrm{el} \mathrm{m}^{-2}$ ). As is well known, the data exhibit diurnal, day-today, and seasonal variability. Fig. 3 shows the variation of the monthly average of TEC (upper curves), and the standard deviation of TEC about the monthly average (lower curves) as a function of time for the four months.

The low solar cycle epoch and the northern geographic location of the subionospheric point are exhibited by the rather low absolute values of the TEC for all reported months. The TEC never exceeded 20 TEC units, and average monthly values of TEC were always below 15 TEC units. The data exhibit diurnal, day-to-day, and seasonal variability. The seasonal variation is exhibited by the time length of the postsunset to dawn minimum TEC values indicating no illumination, and by the progressively lower average day values of the spring (April), summer (August), autumn (October) and winter (December) TEC, respectively.
The low winter values are possibly related to the midlatitude trough [4]; the differences, however, were quite small ( $\sim 1$ to 3 TEC units). This is in contrast to TEC values at midlatitudes (Fort Monmouth, NJ) near solar minimum where summer TEC was higher than winter TEC and both were higher than TEC during the equinoctial periods. No seasonal variability of the day-to-day changes is exhibited by the comparable values of the ratio of the standard deviation to the average TEC values as a function of time. Such ratios are plotted in Fig. 4 for the various months. For the most part, the ratio is below $\sim 25$ percent during the daytime for all reported months. During darkness, the ratio may exceed 25 percent and often exhibits variable periodic behavior. The daytime observations of the ratio agree with those at other locations and other times [5], [6], [7], [8].

By comparison, superposed diurnal variation, monthly average variation and the standard deviation of TEC for the latter half of August 1980, with smoothed observed sunspot number of 150 are shown in Fig. 5. Average maximum monthly values were about double of those during the comparable period of 1977 . While the standard deviation about the monthly average was higher, the daytime ratio of the standard deviation to the TEC (Fig. 5(c)) was comparable with values of $\$ 25$ percent. Late summer TEC data at midlatitudes (Fort Monmouth, NJ) during periods near minimum and maximum solar phase indicate average maximum monthly values more than three times as high at solar maximum as at 


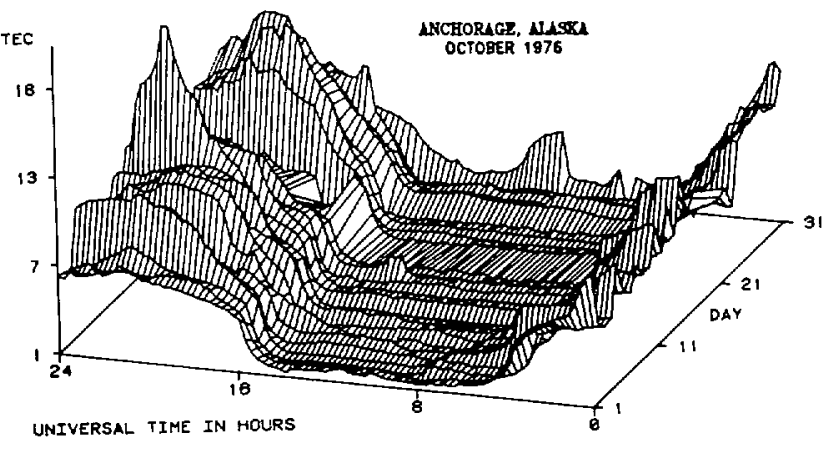

(a)

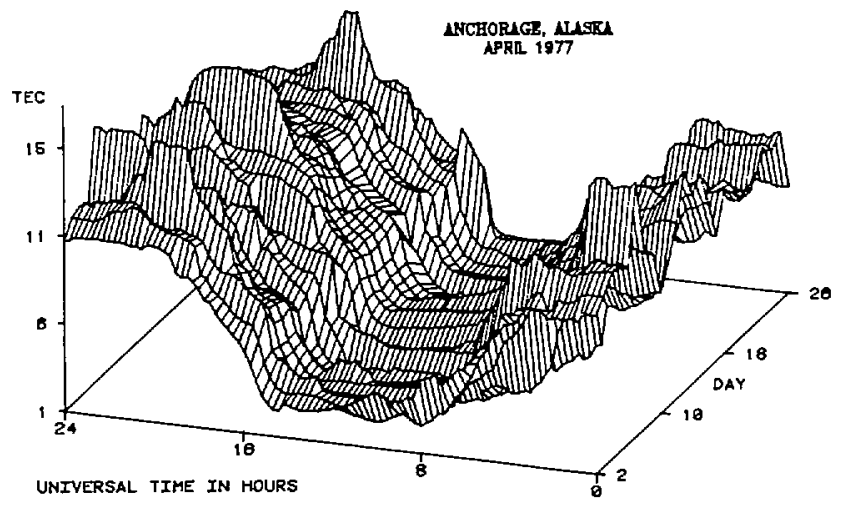

(c)

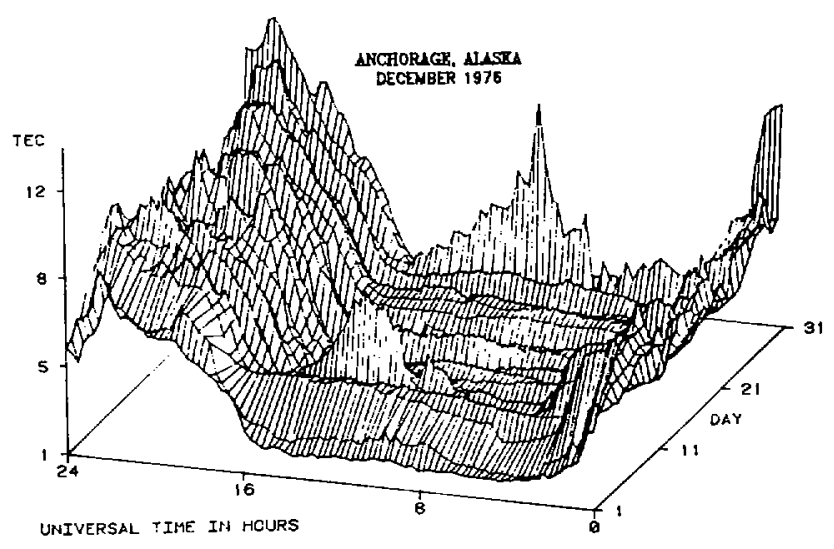

(b)

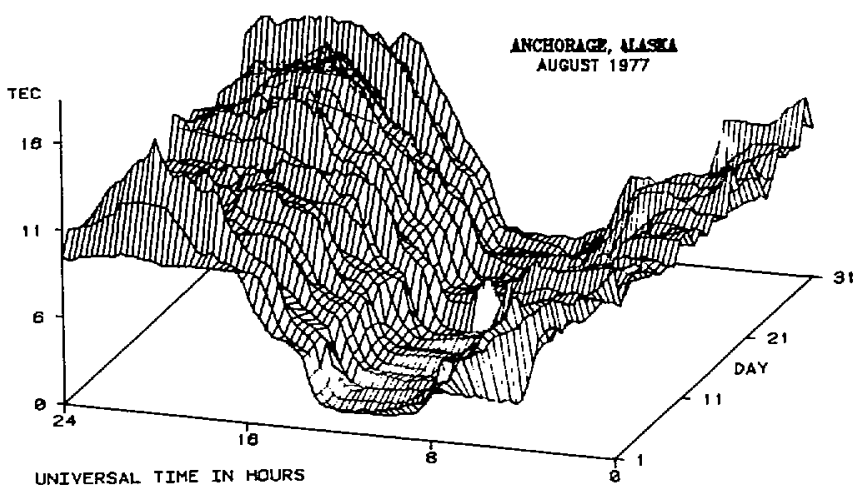

(d)

Fig. 2. Diurnal variation of total electron content in Anchorage, AK as a function of day of month. (a) October 1976. (b) December 1976. (c) April 1977. (d) August 1977.

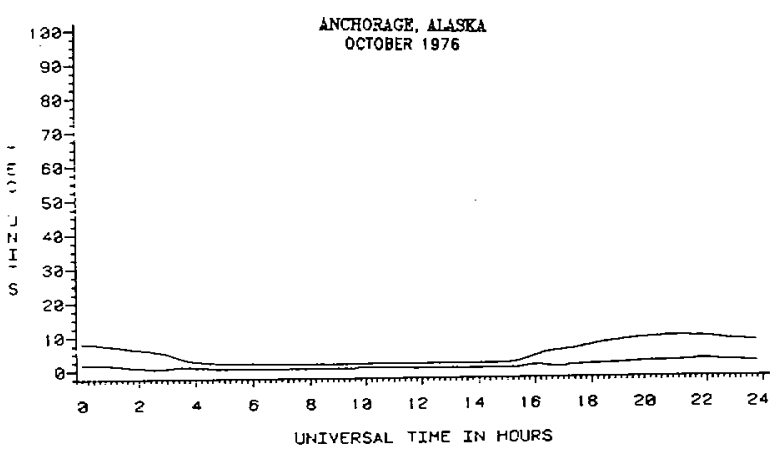

(a)

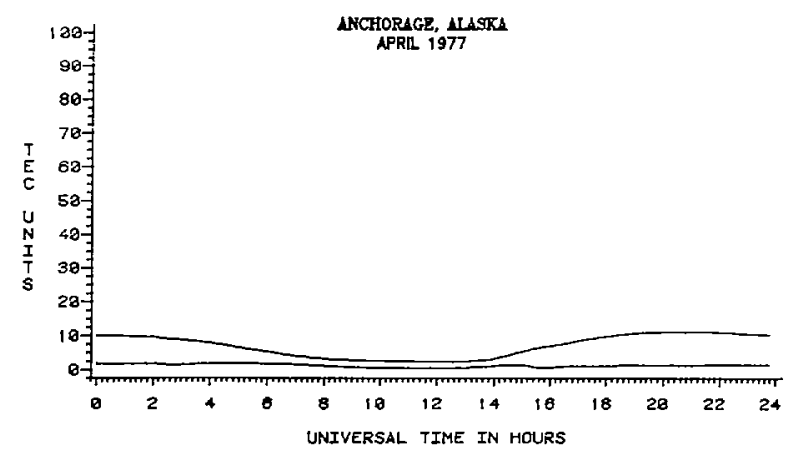

(c)

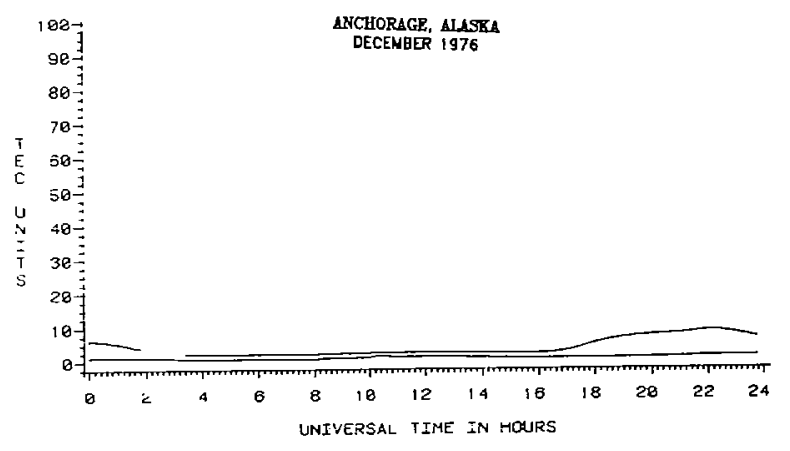

(b)

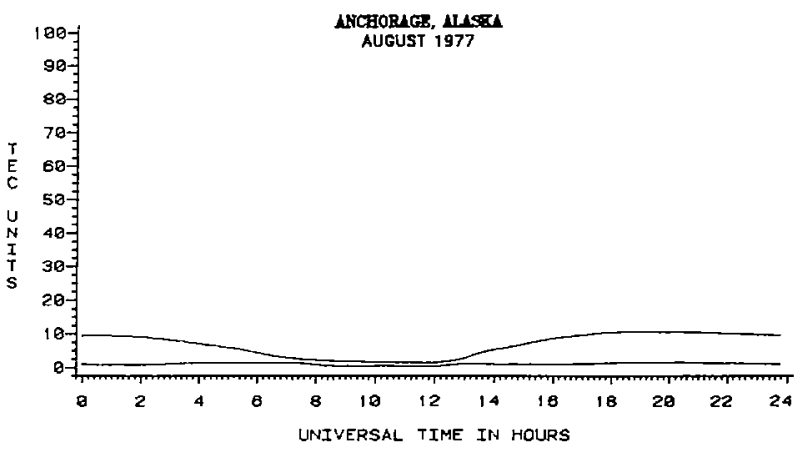

(d)

Fig. 3. Monthly average variation of total electron content-upper curves, and the standard deviation of TEC about the monthly average-lower curves, in Anchorage, AK. (a) October 1976. (b) December 1976. (c) April 1977. (d) August 1977. 


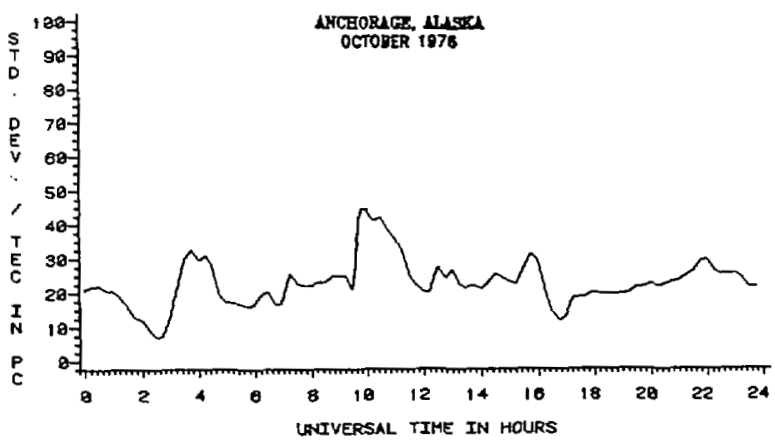

(a)

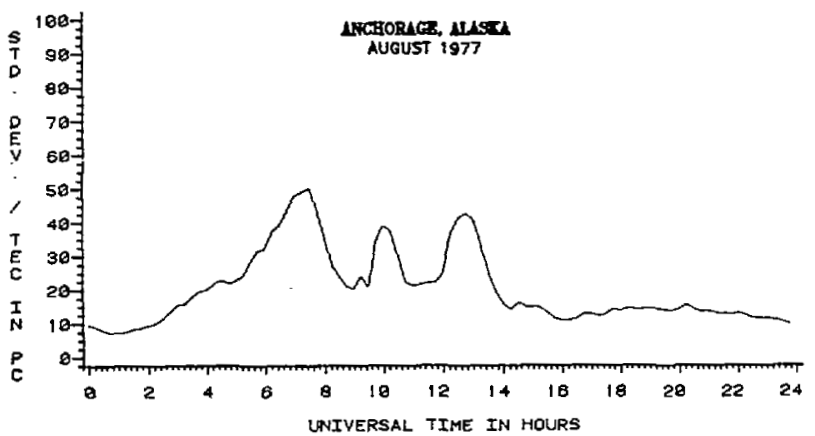

(c)

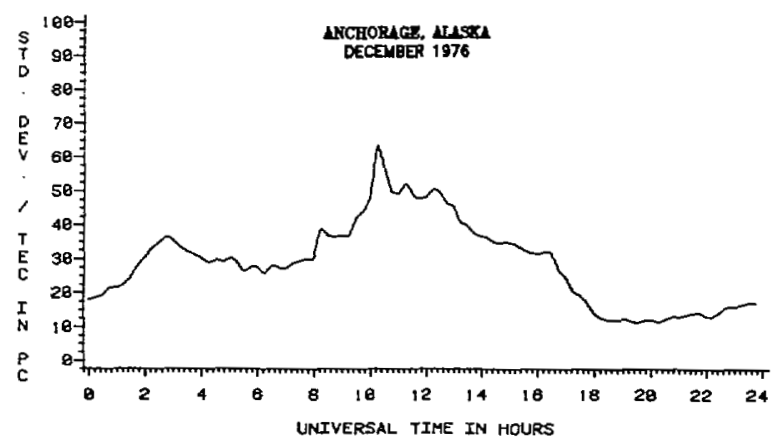

(b)

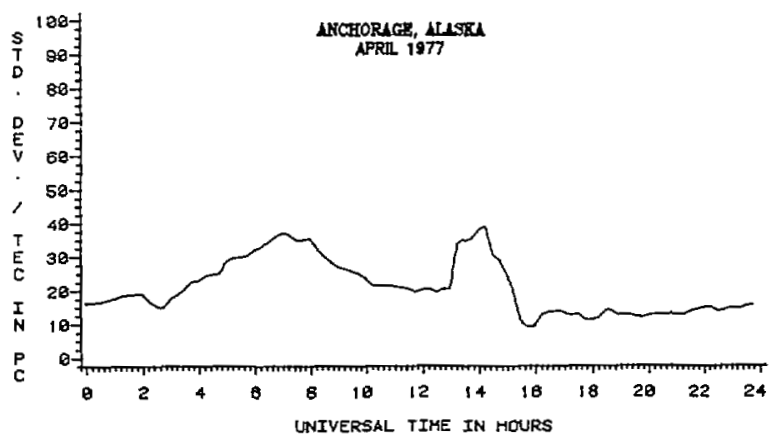

(d)

Fig. 4. The diurnal variation of the ratio (in percent) of the monthly standard deviation of TEC to the average TEC in Anchorage, AK. (a) October 1976. (b) December 1976. (c) April 1977. (d) August 1977.

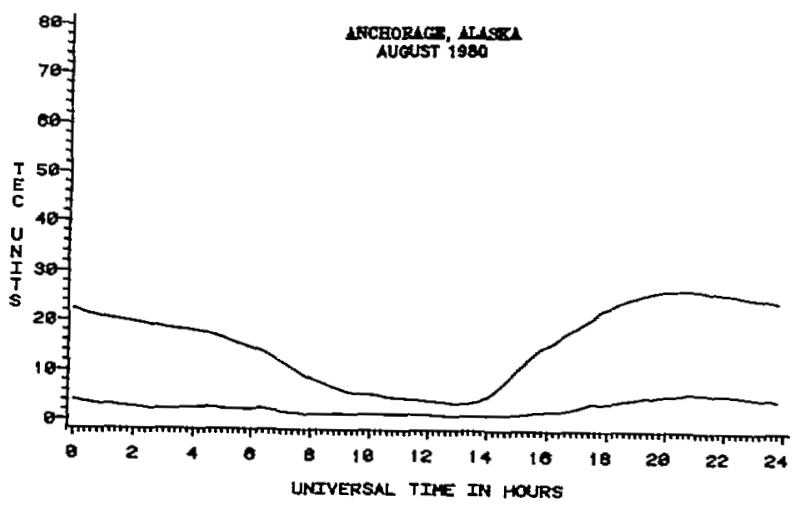

(a)

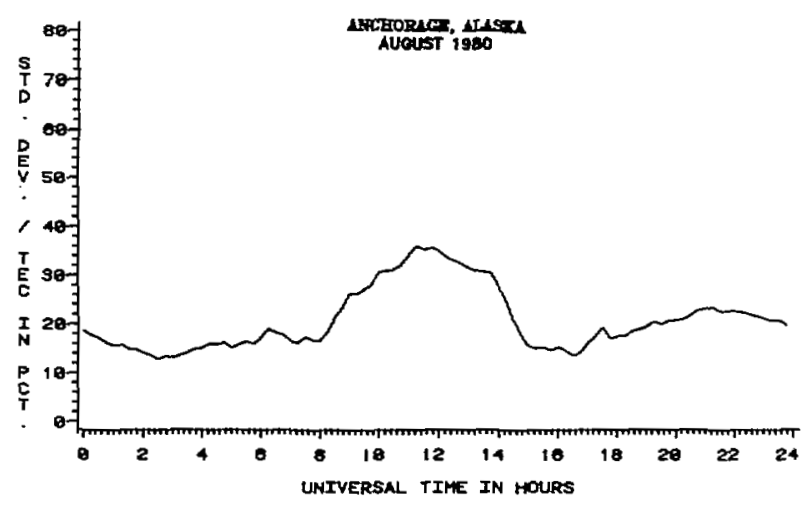

(c)

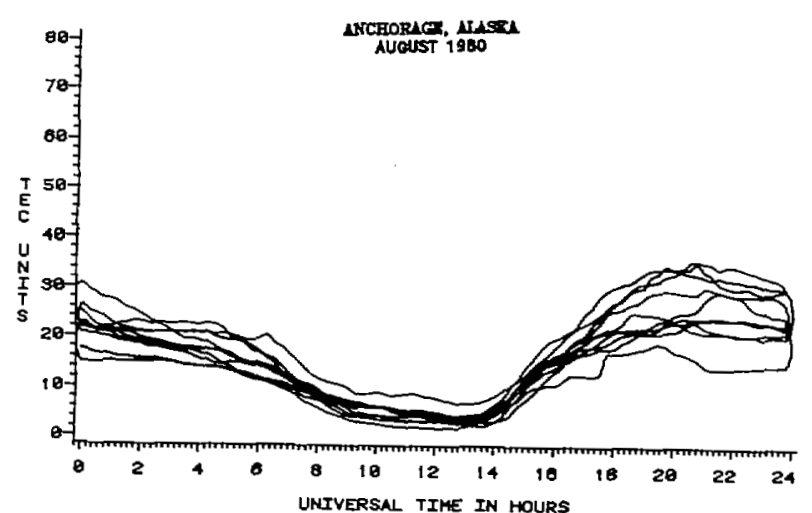

(b)

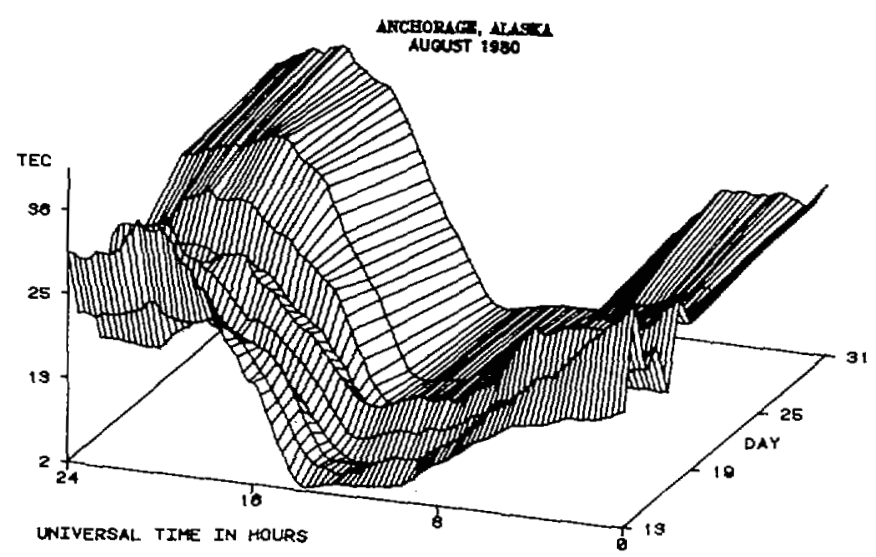

(d)

Fig. 5. TEC data for Anchorage, AK, for August 1980. (a) Monthly average variation of TEC-upper curve, and the standard deviation of TEC about the monthly average-lower curve. (b) Superposed diurnal variation of TEC. (c) Diurnal variation of the ratio (in percent) of the monthly standard deviation to the average TEC. (d) Diurnal variation of TEC as a function of day of month. 


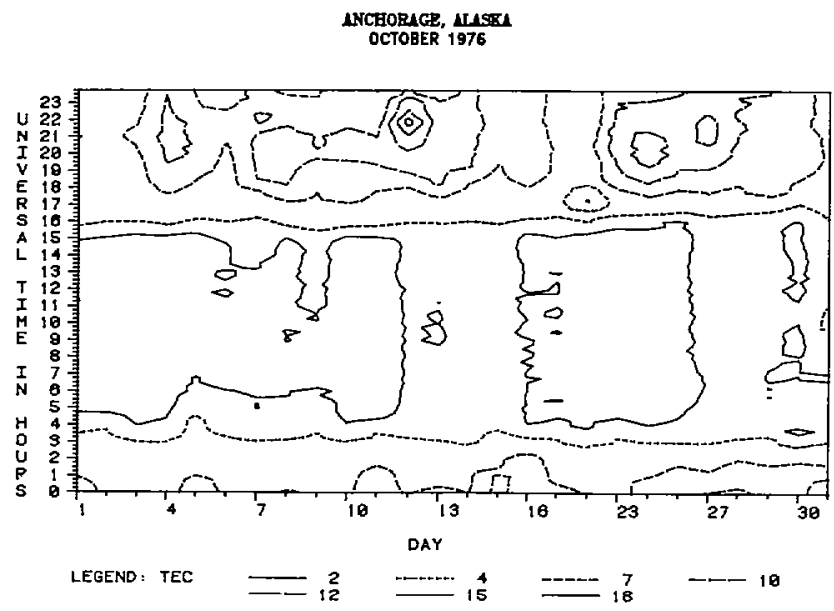

(a)

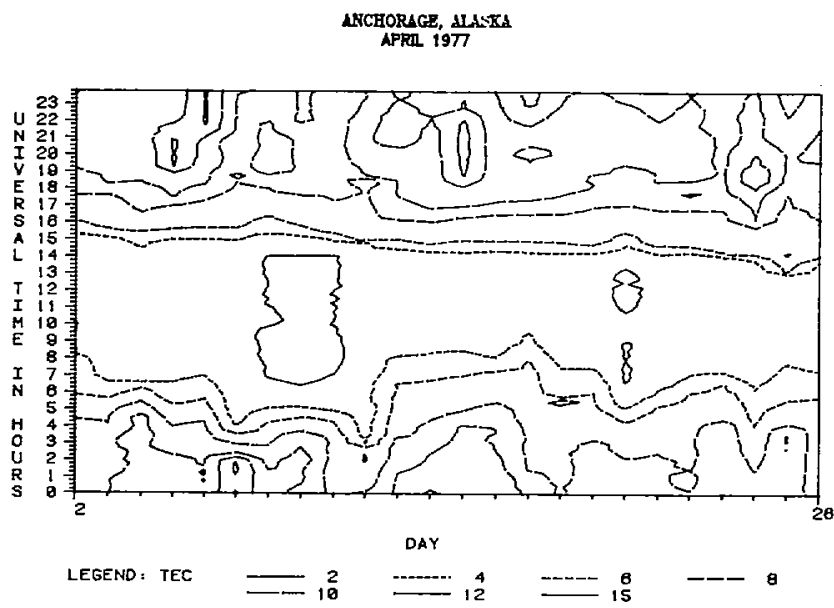

(c)

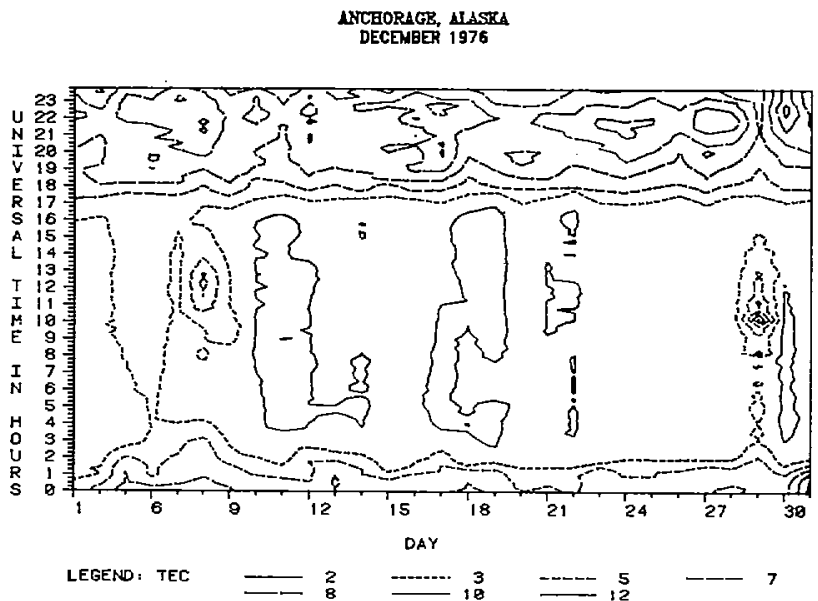

(b)

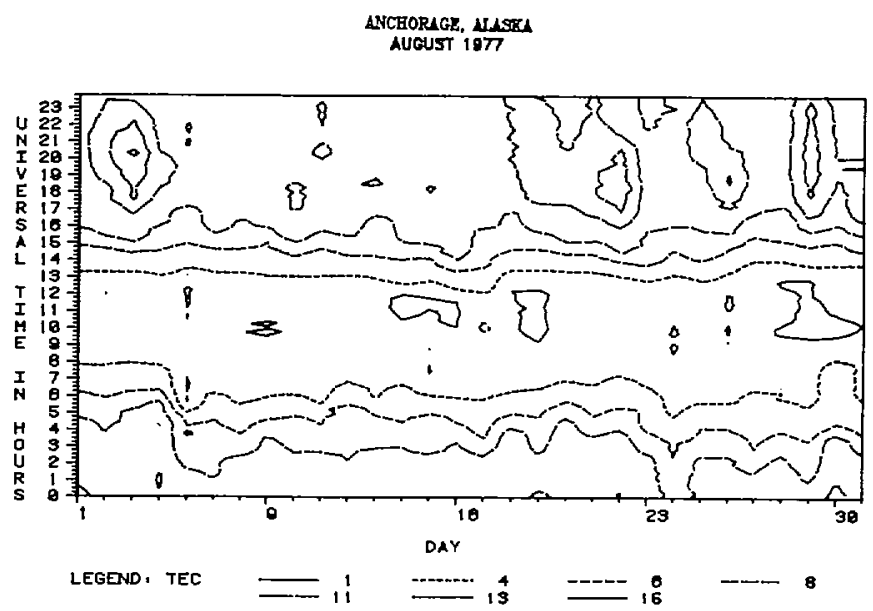

(d)

Fig. 6. Iso-TEC contours as a function of day of month and time in UT at Anchorage, AK. (a) October 1976. (b) December 1976. (c) April 1977. (d) August 1977.

solar minimum. The daytime ratio of the standard deviation to TEC was comparable with values of $\leqslant 25$ percent [9].

A complementary method of assessing the day-to-day variability of TEC is to plot iso-TEC contours as a function of day-of-month and diurnal time. Such plots for the reported months near minimum solar phase are shown in Fig. 6. The values of the intervals of the TEC contours are of the order of the standard deviations, so as to eliminate the appearance of normal day-to-day undulations (e.g. those due to traveling ionospheric disturbances) in these plots.

The following day-to-day features may be ascertained from the figures. For the winter data the iso-TEC contours appear to vary little throughout the month during the buildup (17-19 UT) and final decay (02-04 UT) phases of the diurnal variation. During the maximum phase of the diurnal variation enhanced TEC is normally maintained for a number of consecutive days. Similarly, during the predawn phase depressed TEC values are normally maintained for a number of consecutive days.

The increase in the nighttime TEC on December 29 followed by depressed values on December 30 may be associated with TEC positive phase response to a sudden commencement (SC) which occurred on December 28 at
20:36 UT. No such response during daytime is observed, although enhanced values are observed on December 30 .

For the spring data the iso-TEC contours appear to vary little throughout the month during the buildup (15-17 UT) and final decay (06-08 UT) phases of the diurnal variation. During the maximum phase of the diurnal variation enhanced TEC may be maintained for a number of consecutive days. Similarly, during the predawn phase, depressed TEC values are normally maintained for a number of consecutive days. The low TEC values during the predawn period on April 20 may be due to SC at 0106 on April 19. The April 6-7 period was magnetically disturbed, and this is exhibited by the variability of content during the maximum phase of the diurnal variation.

During the summer little variation of the iso-TEC contours is observed during the decay and buildup phases of the diurnal variation. TEC enhancements/depressions during the day/ night periods normally extend over few consecutive days. One day enhancements/depressions, which do occur, are not correlated with magnetic sudden commencement. (One day enhancements near solar maximum are well correlated with sudden commencement activity on August 15, 16, 17, 1980.) 
During the autumn little variation of the iso-TEC contours is observed during final decay and sunrise period of the diurnal variation. Enhancements/depressions of TEC during maximum/minimum phases of the diurnal variation normally occur for a number of consecutive days. One-day enhancements/ depressions of TEC are not associated with magnetic activity.

While no consistent direct correlation between TEC variability and solar flux/magnetic activity parameters is apparent, for any of the discussed months some variability features may be related. For example, the low predawn values from August 28-31 may be related to the fact that the entire period was magnetically very quiet. The relatively large TEC on the afternoon of August 29 is possibly related to the increased solar flux for that day as indicated by the Ottawa $10.7 \mathrm{~cm}$ flux. However, a lesser TEC on the prior day cannot be related to either parameter.

Faraday rotation is a terrestrial-magnetic-field dependent phenomenon. Since its magnitude is heavily weighted near the earth, it is considered to provide electron content values at altitudes below $1500 \mathrm{~km}$. Signal excess time delay at higher altitudes is due to free electrons in the plasmasphere. Generally, near the minimum of solar phase, the ratio of the plasmaspheric content to the ionospheric (Faraday) content varies from $\leqslant 15$ percent during the day to $\sim 50$ percent during the night [10], [11]. Some diurnal and seasonal variations are observed, but in absolute value the plasma spheric changes are rather small when compared to coincident ionospheric changes. In view of these small relative plasma spheric absolute values and variabilities especially during the day when the effects of ionospheric content in operational considerations are largest, it appears that a simple additive constant value to the ionspheric content of 15 percent of the noontime value may account sufficiently for the plasmaspheric delay [12].

\section{CONCLUSION}

The behavior of TEC at a subauroral latitude location during a period near the minimum phase of the current solar cycle is presented. Seasonal and day-to-day variabilities are observed.

The seasonal variability is seen in the magnitude of the absolute values of TEC maximizing in the spring, minimizing in the winter (possibly due to the midlatitude trough) and being somewhere in between for the summer and autumn. Although absolute differences of TEC are small for the various seasons, these results are in contrast to midlatitude TEC behavior during similar periods. For all seasons the day-to-day behavior of TEC appears uniform during the buildup and decay phases of the diurnal variation, although for the fall period the former is more confined to the sunrise period. TEC enhancements/ depressions during maximum/minimum phases of the diurnal variation are maintained for a few consecutive days during all seasons, but single day enhancements/depressions are often observed. TEC enhancements during the nighttime periods in winter may be associated with positive response to sudden commencement. Similarly, TEC depressions on following nights in winter may be associated with storm behavior of TEC. No daytime TEC response, either positive or negative, has been observed. During the other months magnetic activity was rather subdued. However, no single day enhancement/ depression of TEC appears correlated with magnetic activity. By contrast, single day enhancements during a period near the maximum phase of the solar cycle are correlated with magnetic sudden commencement activity.

The ratio of standard deviation to average TEC is a good indicator for day-to-day variability. For all observed periods the ratio, during the day, is normally $\leqslant 25$ percent. The nighttime ratio is significantly higher, and it often undulates with variable periods.

Current simple prediction models are able to predict monthly median TEC to within an rms of 20-25 percent during the day and 30-35 percent at night [13]. Greater accuracy in day-to-day predictions can probably only be achieved with the use of near real time data at suitable observation stations as a model update. The results presented here, which describe day-to-day patterns of behavior of TEC, have important implications to improvement of currently available models and may lessen the dependence on near realtime data update.

\section{ACKNOWLEDGMENT}

The Faraday observations were performed under the guidance of Mr. F. J. Gorman, U.S. Army, Communications/ Automatic Data Processing Center, Fort Monmouth, NJ. The analysis of the raw data and basic computations were performed under the guidance of Mr. W. Chaffee, Monmouth College, West Long Branch, NJ.

\section{REFERENCES}

[1] J. A. Klobuchar, "Total electron content studies of the ionosphere," AGARD-AG-166 on Total Electron Content and Scintillation Studies of the Ionosphere," J. Aarons, Ed., Mar. 1973.

[2] P. A. Hicks, "A low cost, all electronic Faraday rotation polarimeter," presented at COSPAR Symp. Future Appl. of Satellite Beacon Measurement, Comm. Space Res., Graz, Austria, 1972.

[3] J. A. Klobuchar, "Polarization of AZ-E1 mounted antenna viewing celestial objects," IEEE Trans. Antennas Propagat., vol. AP-14, p. $650,1966$.

[4] D. B. Muldrew, "F-layer ionization troughs deduced from Alouette data," J. Geophys. Res., vol. 70, pp. 2635-2650, 1965.

[5] H. Soicher and F. J. Gorman, "Variability of total electron content at temperate and high latitudes," Proc. COSPAR/URSI Symp. Sci. Eng. Uses of Satellite Radio Beacons, 1980, pp. 91-98.

[6] H. Soicher, Z. Houminer, and A. Shuval, "Total electron content structure in the Middle East," Radio Sci., vol. 17, no. 6, pp. 1623$1631,1982$.

[7] H. Soicher, J. A. Klobuchar, and P. H. Doherty, "Spatial variability of total electron content in the eastern Mediterranean region," Radio Sci., vol. 19, no. 3, pp. 757-764, 1984.

[8] H. Soicher and F. J. Gorman, "Variability of total electron content at temperate and high latitudes," Proc. COSPAR/URSI Symp. Sci. Eng. Uses of Satellite Radio Beacons, A. Wernik, Ed. WarsawLodz, Polish Scientific Publisher, 1981, pp. 91-97.

[9] - - "Seasonal and day-to-day variability of total electron content at midlatitudes near solar maximum," Radio Sci., vol. 20, no. 3, pp. 383-387, 1985.

[10] H. Soicher, "Ionospheric and plasmaspheric effects in satellite navigation systems," IEEE Trans. Antennas Propagat., vol. AP-25, no. 5, pp. 705-708, 1977.

[11] K. Davies, "Recent progress in satellite radio beacon studies with particular emphasis on the ATS-6 radio beacon experiment," Space Sci. Rev., vol. 25, pp. 357-430, 1980.

[12] _- " "Review of recent progress in ionospheric predictions," Radio Sci., vol. 16, no. 6, pp. 1407-1430, Nov-Dec., 1981.

[13] CCIR Rep. 725-1, "Ionospheric properties," Recommendations and Reports of the CCIR, 1982, vol. VI, Geneva, 1982. 


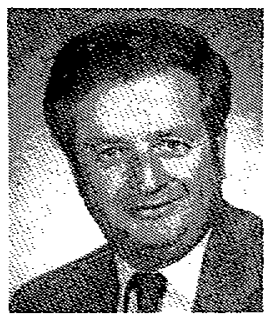

Haim Soicher received the B.S. degree in mathematics and physics from Brooklyn College, Brooklyn, NY, in 1959, the M.S. and Ph.D. degrees in physics from New York University, New York, NY, in 1961 and 1972, respectively, and the M.B.A. degree from Fairleigh Dickinson University, Rutherford, NJ, in 1979.

Since 1960 he has been at the Communications/ Automatic Data Processing Center and its predecessor organizations at the U.S. Army Communications-Electronics Command, Fort Monmouth, NJ.
He has been engaged in radio wave propagation studies and ionospheric research.

Dr. Soicher is currently Chairman of the Center's Research Council; Chairman of the Electromagnetic Wave Propagation Panel, NATO Advisory Group for Aerospace Research and Development (AGARD); Associate Editor of Radio Science; principal U.S. Army member of the U.S. National Committee of CCIR Study Group 6: and member Commission G of URSI. He has published more than 50 papers in the open literature, chaired and cochaired five national/international symposia and edited/co-edited their proceedings.

\title{
Hybrid Solutions for Large-Impedance Coated Bodies of Revolution
}

\author{
LOUIS N. MEDGYESI-MITSCHANG, SENIOR MEMBER, IEEE, AND DAU-SING WANG, MEMBER, IEEE
}

\begin{abstract}
Electromagnetic scattering solutions are developed for coated perfectly conducting bodies of revolution (BOR) that satisfy the impedance boundary condition. The integral equation arising from the impedance (Leontovich) boundary condition is solved by use of the method of moments (MM) technique along with an Ansatz for the surface currents that is derived from physical optics (PO) and the Fock theory that is modified for imperfectly conducting surfaces. The MM solution is expressed in terms of two integral (Galerkin) operators. The form of the Galerkin expansion used results in a symmetric MM system matrix. The hybrid solution is specialized for BOR's although the approach is applicable to a broader class of scatterers as well. The results are compared with the Mie solution for penetrable spherical scatterers, which satisfy the impedance boundary condition, and with recently published MM solutions for nonspherical scatterers.
\end{abstract}

\section{INTRODUCTION}

W IDESPREAD USE OF composite materials has renewed interest in electromagnetic effects associated with imperfectly conducting bodies. Prominent fundamental findings were established in this area by a number of early investigators, among them Leontovich and Fock [1]-[3]. Later, Senior [4] examined the implications of the impedance (Leontovich) boundary in mathematical as well as applied contexts. Alexopoulos and Tadler investigated the efficacy of the impedance (Leontovich) boundary condition (IBC) for discontinuous surface impedances and composite scatterers [5]-[7]. Subsequently, Wait and his co-workers addressed the

Manuscript received February 3, 1986; revised May 8, 1986. This work was conducted under McDonnell Douglas Independent Research and Development program.

The authors are with the McDonnell Douglas Research Laboratories, St. Louis, MO 63166

IEEE Log Number 8610020 . effects of imperfectly conducting surfaces and boundaries in radio propagation, navigation, and applied geophysics [8][13].

In a series of classic papers [3], Fock outlined a new approach, called the parabolic equation method, to solve electromagnetic scattering problems involving classes of perfectly and imperfectly conducting surfaces. He showed that the elliptic equations describing wave motion could be transformed into parabolic ones, if one assumes wave propagation in a specified direction and that the field variation along the surface is slow compared to that of fields normal to the surface. This formulation and its solution, called the Fock theory, has been extended to imperfectly conducting surfaces, satisfying the IBC. In this approach the electrical/material properties of the penetrable boundary are characterized by a surface impedance. The radii of curvature at all points on the surface are assumed to be large in comparison to wavelength $(\lambda)$ and the skin depth is small. Bowman and Weston [14] demonstrated the surface impedance characterization for multilayered cylindrical and spherical scatterers.

In the present investigation the Fock results as an Ansatz are combined with the method of moments (MM) to obtain hybrid solutions for electrically large, shaped bodies which satisfy the IBC. This approach parallels an earlier hybrid analysis for electrically large, perfectly conducting bodies (specifically, BOR's) that are not amenable to the geometrical or physical theory of diffraction (GTD or PTD) alone because of their geometry, nor to the MM technique because of their electrical size [15]. As shown in [15], the principal advantage of using the Fock Ansatz is that it yields the surface currents on the scatterer and thus can be incorporated directly into an electric 